\title{
Right-Sided Diverticulosis and Disparities from Left-Sided Diverticulosis in the Vietnamese Population Living in Boston, Mass., USA: A Retrospective Cohort Study
}

\author{
Raman Mehrzad $^{\mathrm{a}}$ Suprav Mishra ${ }^{\mathrm{a}}$ Garrey Faller $^{\mathrm{b}}$ Babar Memon $^{\mathrm{a}}$ \\ Joseph Fiore ${ }^{c}$
}

Departments of a Internal Medicine, ${ }^{\mathrm{b}}$ Pathology and ${ }^{\mathrm{c}}$ Medicine, Division of Gastroenterology, Steward Carney

Hospital, Boston, Mass., USA

\section{Key Words}

Diverticulosis · Prevalence · Vietnamese $\cdot$ Complications

\begin{abstract}
Objective: To report the prevalence, clinical differences and complications of right-sided diverticulosis (RD) and to investigate the potential disparities from left-sided diverticulosis (LD) in the Vietnamese population. Subjects and Methods: A retrospective cohort study was conducted using medical records of Vietnamese-born patients from 2000 to 2013 in a community teaching hospital in Boston, Mass., USA. By simple randomization, a randomized control group of 299 Caucasian patients was also selected from the same time frame [167 males (M) and 132 females (F)]. Colonoscopy reports were reviewed for demographics (age and gender), indication and anatomical location of the colonic diverticulosis (CD), concomitant colonic findings, symptoms, and endoscopic complications. Results: A total of 207 patients were included in the Vietnamese cohort (mean age $61.6 \pm 8.9$ years). The mean age at first screening colonoscopy was 58.2 \pm 7.2 years (114 F/92 M, 55.7/44.4\%). Our study identified 104 (50.5\%) patients with LD (57 M/47 F), 65 (31.1\%) with RD (35 $\mathrm{M} / 30 \mathrm{~F}$ ) and 38 (18.4\%) with both LD and RD (23 M/15 F); 133 (64\%) were asymptomatic. A total of 21 (33\%) patients with $\mathrm{RD}$ were symptomatic. The mean age of the control group
\end{abstract}

\begin{tabular}{ll}
\hline KARGER 125/s & $\begin{array}{l}\text { ( } 2015 \text { S. Karger AG, Basel } \\
1011-7571 / 15 / 0244-0355 \$ 39.50 / 0\end{array}$ \\
$\begin{array}{l}\text { E-Mail karger@karger.com } \\
\text { www.karger.com/mpp }\end{array}$ & $\begin{array}{l}\text { This is an Open Access article licensed under the terms of the } \\
\text { Creative Commons Attribution-NonCommercial } 3.0 \text { Un- } \\
\text { ported license (CC BY-NC) (www.karger.com/OA-license), } \\
\text { applicable to the online version of the article only. Distribu- } \\
\text { tion permitted for non-commercial purposes only. }\end{array}$
\end{tabular}

was $61.6 \pm 8.1$ years. The average age at first screening colonoscopy was $52.8 \pm 6.4$ years. Of the 299 in the Caucasian group, 254 (84.9\%) had LD (114 M/140 F), 9 (3.0\%) had RD ( $2 \mathrm{M} / 7 \mathrm{~F})$ and 36 (12\%) had both LD and RD (16 M/20 F); 225 (75\%) were asymptomatic and came in for screening colonoscopies. A total of 2 patients (22\%) with RD were symptomatic. Conclusion: RD was common in this Vietnamese population, and the prevalence was higher than in the Caucasian control group.

(c) 2015 S. Karger AG, Basel

\section{Introduction}

Colonic diverticulosis (CD) is a common condition with a rising prevalence worldwide [1-3]. The prevalence varies with national origin, cultural background and diet and is increasing over time and with advanced age. It is estimated that $30 \%$ of the population at age 50 and $50 \%$ after the age of 70 have CD [4-6]. CD is generally an acquired disease whose pathophysiology is complex and still not fully understood [7]. Risk factors such as colonic immobility, changes in the colonic wall, low-grade inflammation, and an imbalance in microflora contribute to the development of diverticula [8]. Diverticular disease (DD) of the colon refers to symptomatic diverticula that
Raman Mehrzad, MD Steward Carney Hospital 2100 Dorchester Ave.

Boston, MA 02169 (USA)

E-Mail raman_m1@ hotmail.com 
cause complications such as acute diverticulitis, perforation and lower gastrointestinal (GI) bleeding, the latter being the most common cause of all GI bleeding [9-11]. An estimated $20 \%$ of patients with CD will develop symptoms throughout their lifespan, and approximately $1-2 \%$ will require hospitalization, with $0.5 \%$ needing surgical treatment [12].

Although CD can develop anywhere in the colon, right-sided diverticulosis (RD) is uncommon and reported to occur in 1-2\% of surgical specimens in Europe and America. While RD is more common in most areas of Asia $[4,13]$, the prevalence in the Vietnamese population is unknown. With an increasing prevalence, unclear etiology and potential dangerous complications, understanding the true prevalence in different populations is important. The aim of this study was to report the prevalence, clinical features and complications of RD and to investigate the potential disparities from left-sided diverticulosis (LD) in the Vietnamese population living in the area of Boston, Mass., USA.

\section{Subjects and Methods}

\section{Data Collection}

A retrospective cohort study was conducted using medical records of Vietnamese-born patients from 2000 to 2013 in a community teaching hospital (Steward Carney Hospital) in Boston, Mass., USA. By simple randomization, a randomized control group of 299 Caucasian patients was also selected from the same time frame. Colonoscopy reports were reviewed for demographics (age and gender), indication and anatomical location of the CD, concomitant colonic findings, symptoms, and endoscopic complications. Symptomatic indications for colonoscopy in patients who could potentially develop DD of the colon were abdominal pain, GI bleeding, bloating, and changes in bowel movements (constipation and/or diarrhea). Endoscopic complications were defined as perforation, postprocedural infection, hemorrhage, abdominal pain, cardiopulmonary complications, and death. Both groups were analyzed separately and were subsequently compared. An independent $t$ test was used to determine whether there was a statistically significant difference between the mean values of the Vietnamese cohort and the Caucasian controls. A $\chi^{2}$ test was used to determine whether there was a significant difference between the expected and the observed frequencies.

\section{Inclusion Criteria}

All Vietnamese-born patients who underwent colonoscopy with the diagnosis of diverticulosis at our community teaching hospital from 2000 to 2013 were included as well as a randomized control group of Caucasian patients during the same time frame. Diagnosis of CD was defined as two or more diverticula in one or more sites of the bowel identified with colonoscopy. RD was defined as diverticulosis in the cecum, ascending colon and/or hepatic flexure, whereas LD was defined as diverticulosis in the splenic flexure, descending colon and/or sigmoid colon. DD of the colon was defined as perforation, lower GI bleeding and diverticulitis.

\section{Histology}

All colonic biopsies from the colonoscopies and resections were collected. The pathology reports from all biopsies and resections were reviewed (by G.F.), and the slides from the 3 colonic resections were also reviewed to evaluate for any possible histopathological differences.

\section{Results}

A total of 506 patients were included in our study. Of these, 207 Vietnamese patients met the inclusion criteria, and 299 Caucasians patients were randomly selected as controls.

\section{Vietnamese Patients}

The mean age was $61.6 \pm 8.9$ years and the mean age at first screening colonoscopy was $58.2 \pm 7.2$ years. There were $114(55.7 \%)$ females (F) and $92(44.4 \%)$ males (M). Our study identified $104(50.5 \%)$ patients with LD (57 $\mathrm{M} / 47 \mathrm{~F}), 65$ (31.1\%) with RD (35 M/30 F) and 38 (18.4\%) with both $\mathrm{LD}$ and $\mathrm{RD}(23 \mathrm{M} / 15 \mathrm{~F})$. The anatomical distribution of CD was as follows: 49 in the cecum; 95 in the ascending colon; 19 in the transverse colon; 98 in the descending colon, and 111 in the sigmoid colon.

Of the 207 Vietnamese patients, 133 (64\%) were asymptomatic and came in for screening colonoscopies. A total of 74 (36\%) patients were symptomatic. Of these, 21 (28.3\%) were RD, 38 (51.4\%) LD and 15 (20.7\%) both $\mathrm{RD}$ and $\mathrm{LD}$. Symptom distribution for $\mathrm{RD}$ was as follows: bleeding: 14 patients; abdominal pain: 7 patients, and changes in bowel movements: 2 patients; 3 patients had diverticulitis. There were no endoscopic complications. Symptom distribution for LD was as follows: bleeding: 32 patients; abdominal pain: 9 patients, and changes in bowel movements: 5 patients; 2 patients had diverticulitis. There were no endoscopic complications. In patients with CD on both the left and right sides, 15 (39\%) patients were symptomatic. Symptom distribution was as follows: bleeding: 10 patients; abdominal pain: 2 patients, and changes in bowel movements: 3 patients. There were no endoscopic complications.

Other colonic findings were the following: associated hemorrhoids: 148 (72\%) patients and polyps: 12 patients. Less common colonoscopy findings included lipomas: 2 patients ( 1 at the hepatic flexure and 1 in the cecum); malignancy: 1 patient (adenocarcinoma); hypertrophic anal 
papilla: 1 patient; torturous sigmoid colon: 1 patient, and tubular rectum: 1 patient.

\section{Randomized Control Group}

The mean age was $61.6 \pm 8.1$ years and age at first screening colonoscopy was $52.8 \pm 6.4$ years $(114 \mathrm{M} / 140 \mathrm{~F}$, 44.9/55.1\%). There were $254(84.9 \%)$ patients with LD (114 $\mathrm{M} / 140 \mathrm{~F}), 9(3.0 \%)$ with $\mathrm{RD}(2 \mathrm{M} / 7 \mathrm{~F})$ and $36(12 \%)$ with both $\mathrm{LD}$ and $\mathrm{RD}(16 \mathrm{M} / 20 \mathrm{~F})$. The anatomical distribution of CD was as follows: 21 in the cecum; 32 in the ascending colon; 25 in the transverse colon; 95 in the descending colon, and 212 in the sigmoid colon. Of the 299 patients, 225 (75\%) were asymptomatic and came in for screening colonoscopies and 74 (25\%) were symptomatic. A total of 2 (22\%) patients with RD were symptomatic, with bleeding in 1 patient and abdominal pain in another. There were no associated endoscopic complications. Overall, 62 (24\%) patients with LD were symptomatic. Symptom distribution was as follows: bleeding: 51 patients; abdominal pain: 12 patients, and changes in bowel movements: 6 patients; 2 patients had diverticulitis. There were no associated endoscopic complications. In patients with $\mathrm{CD}$ on both the left and right sides, 10 (28\%) patients were symptomatic. Symptom distribution was as follows: bleeding: 4 patients; abdominal pain: 2 patients; changes in bowel movements: 5 patients, and abdominal bloating: 1 patient. There were no associated endoscopic complications.

Other colonic findings were the following: associated hemorrhoids: 166 (56\%) patients; polyps: 35 patients, and malignancies: 2 patients (cecal adenocarcinoma). Less common colonoscopy findings included stricture: $1 \mathrm{pa}-$ tient; stenosis: 3 patients; lipoma: 3 patients; anal fissures: 2 patients, and lymphoid hypertrophy: 1 patient. Details are presented in table 1.

\section{Statistical Analysis}

There was a statistically significant difference with LD versus $\mathrm{RD}$ and symptom distribution in the Vietnamese and controls groups $(p=0.0001)$. With regard to other colonic findings, there was a statistical difference with hemorrhoids in both cohorts $(\mathrm{p}=0.0001)$ but not with polyps $(\mathrm{p}=0.0648)$.

\section{Histological Specimens}

Of the 3 colonic resections, 1 (from the Vietnamese group) was for a colon adenocarcinoma, and this resection did not contain any diverticula. The diverticula present in the 2 remaining resections (from the Caucasian group) showed the usual outpouchings of colonic mucosa and submucosa through weakness of the muscular
Table 1. Results of our cohort

\begin{tabular}{|c|c|c|}
\hline & $\begin{array}{l}\text { Vietnamese } \\
(\mathrm{n}=207)\end{array}$ & $\begin{array}{l}\text { Controls } \\
(\mathrm{n}=299)\end{array}$ \\
\hline Mean age, years & $61.6 \pm 8.9$ & $61.6 \pm 8.1$ \\
\hline \multicolumn{3}{|l|}{ Age at first colonoscopy, } \\
\hline years & $58.2 \pm 7.2$ & $52.8 \pm 6.4$ \\
\hline Sex predominance & $\begin{array}{l}55 \% \mathrm{~F} \\
(92 \mathrm{M} / 114 \mathrm{~F})\end{array}$ & $\begin{array}{l}56 \% \mathrm{M} \\
(114 \mathrm{M} / 140 \mathrm{~F})\end{array}$ \\
\hline Symptomatic & $74(36 \%)$ & $74(25 \%)$ \\
\hline Asymptomatic & $133(64 \%)$ & $225(75 \%)$ \\
\hline \multicolumn{3}{|l|}{ Location } \\
\hline Cecum & 49 & 21 \\
\hline Ascending colon & 95 & 32 \\
\hline Transverse colon & 19 & 25 \\
\hline Descending colon & 98 & 95 \\
\hline Sigmoid colon & 111 & 212 \\
\hline \multicolumn{3}{|l|}{ Colonoscopy findings } \\
\hline Associated polyps & 12 & 35 \\
\hline Hemorrhoids & $148(72 \%)$ & $166(56 \%)$ \\
\hline Malignancy & 1 & 2 \\
\hline Stricture & 0 & 1 \\
\hline Stenosis & 0 & 3 \\
\hline Lipoma & 2 & 3 \\
\hline Anal fissure & 0 & 2 \\
\hline Lymphoid hypertrophy & 0 & 1 \\
\hline Tubular rectum & 1 & 0 \\
\hline Tortuous sigmoid & 1 & 0 \\
\hline Hypertrophic anal papilla & 1 & 0 \\
\hline
\end{tabular}

\begin{tabular}{lll}
\hline $\begin{array}{l}\text { Distribution } \\
\text { LD }\end{array}$ & & \\
$\quad$ Patients & $104(50.5 \%)$ & $254(84.9 \%)$ \\
$\quad$ Sex predominance & $57 \mathrm{M} / 47 \mathrm{~F}$ & $114 \mathrm{M} / 140 \mathrm{~F}$ \\
Symptomatic & $38(51.4 \%)$ & $62(24 \%)$ \\
Bleeding & 32 & 51 \\
Abdominal pain & 9 & 12 \\
Change in BM & 5 & 6 \\
Abdominal bloating & 0 & 0 \\
RD & & \\
Patients & $65(31.1 \%)$ & $9(3 \%)$ \\
Sex predominance & $34 \mathrm{M} / 30 \mathrm{~F}$ & $2 \mathrm{M} / 7 \mathrm{~F}$ \\
Symptomatic & $21(28.3 \%)$ & $2(22 \%)$ \\
Bleeding & 14 & 1 \\
Abdominal pain & 7 & 1 \\
Change in BM & 2 & 0 \\
Abdominal bloating & 0 & 0 \\
LD + RD & & \\
Patients & $38(18.4 \%)$ & $36(12 \%)$ \\
Sex predominance & $23 \mathrm{M} / 15 \mathrm{~F}$ & $16 \mathrm{M} / 20 \mathrm{~F}$ \\
Symptomatic & $15(20.7 \%)$ & $10(28 \%)$ \\
Bleeding & 10 & 4 \\
Abdominal pain & 2 & 2 \\
Change in BM & 3 & 5 \\
Abdominal bloating & 0 & 1 \\
\hline
\end{tabular}

$\mathrm{BM}=$ Bowel movement. 
Fig. 1. Low-power image of a right-sided diverticulum. HE. It shows a diverticulum with outpouching of the mucosa and submucosa with an attenuated layer of muscle. There is minimal associated chronic inflammation.

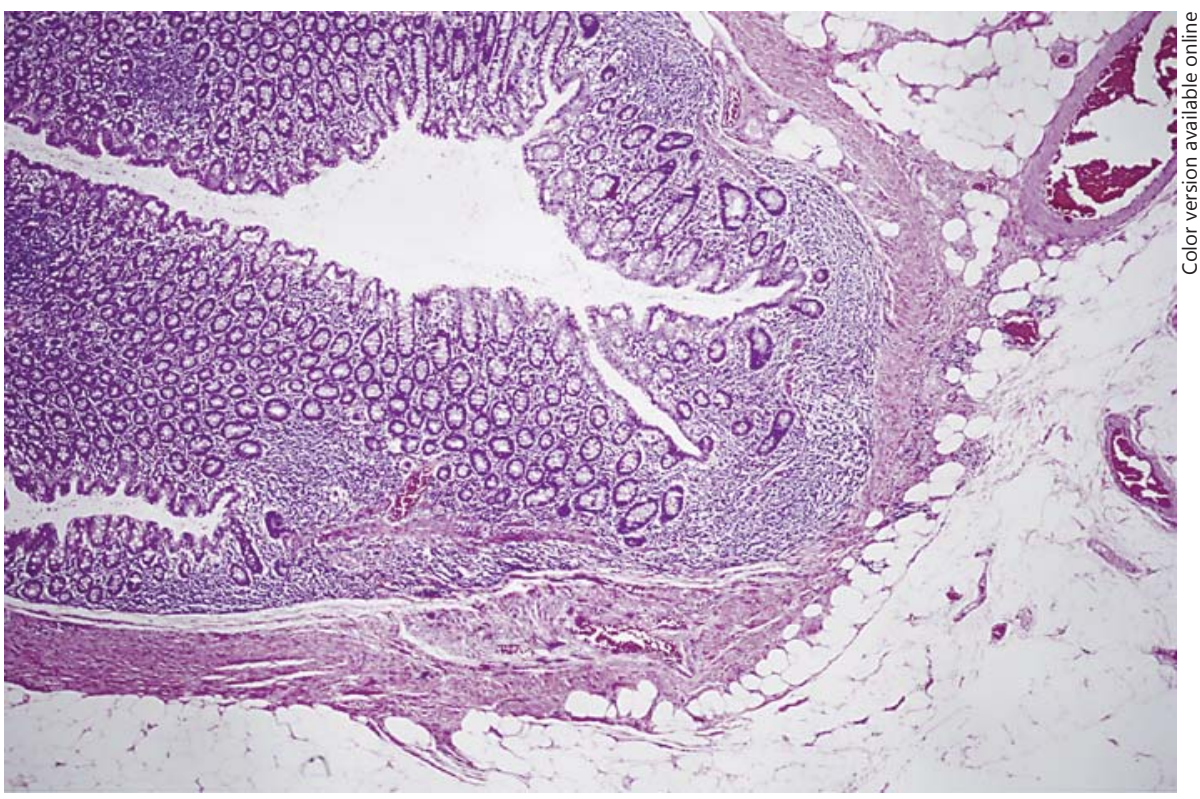

Fig. 2. Medium-power image of a right-sided diverticulum. HE. It shows the diverticulum with outpouching of the mucosa and submucosa with an attenuated layer of muscle.

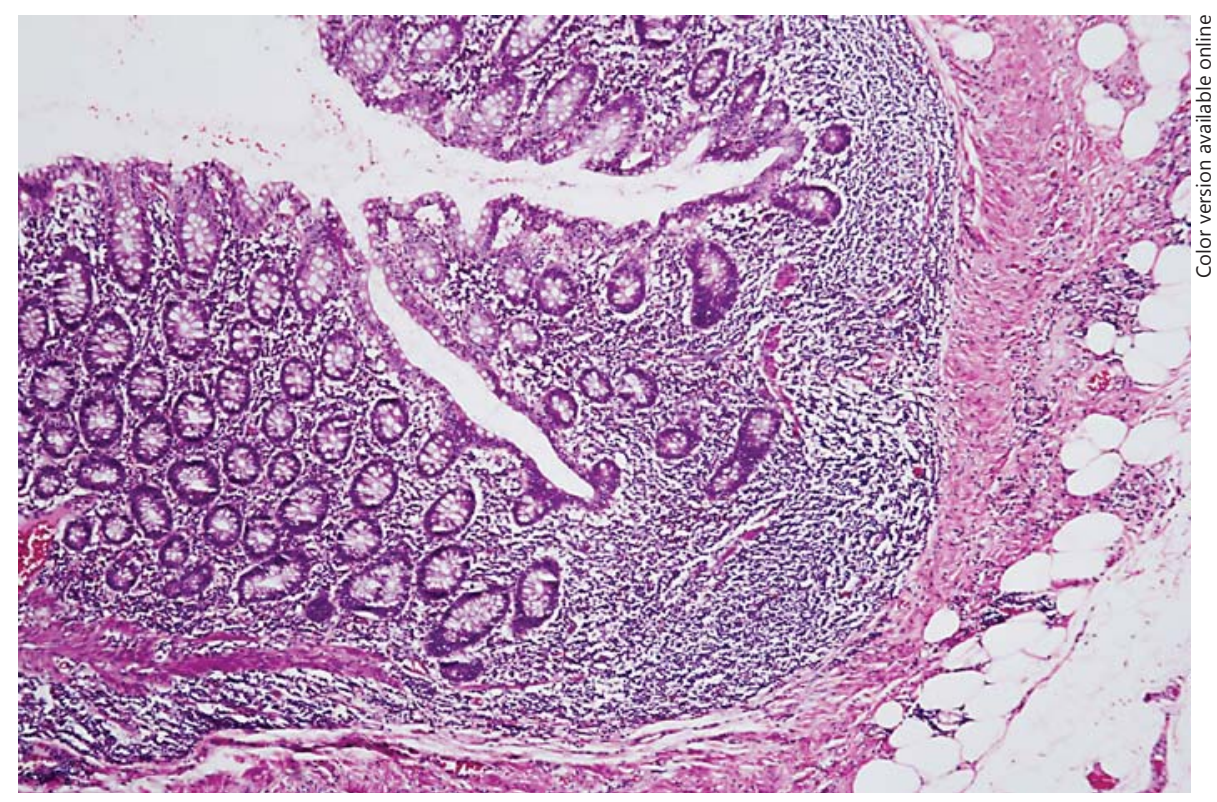

layer of the colonic wall. Some of these were inflamed. No gross anatomical or histological differences were noted with these left-sided diverticula compared to the more common right-sided ones (fig. 1-4).

\section{Discussion}

Our findings showed that $\mathrm{RD}$ was common in the Vietnamese population, and the prevalence was higher than in Caucasians in our study. CD is a common GI dis- order worldwide, with an estimated prevalence of $50 \%$ after the age of $70[5,6,14]$. The true prevalence is, presumably, higher given that most affected individuals are asymptomatic and that screening colonoscopy is not mandatory - nor is it widely available in all countries. The anatomical distribution pattern of CD differs between populations. While $\mathrm{LD}$ is most common worldwide, the prevalence of $\mathrm{RD}$ is higher in the Asian population $[4,15$, 16]. Our study supports that the distribution of $\mathrm{CD}$ in the Vietnamese population follows this pattern $(31 \% \mathrm{RD}$ in Vietnamese vs. $3 \%$ in controls). 
Fig. 3. Low-power image of a left-sided sigmoid diverticulum for comparison. HE. It shows a diverticulum with outpouching of the mucosa and submucosa with an attenuated layer of muscle. There is associated acute and chronic inflammation.

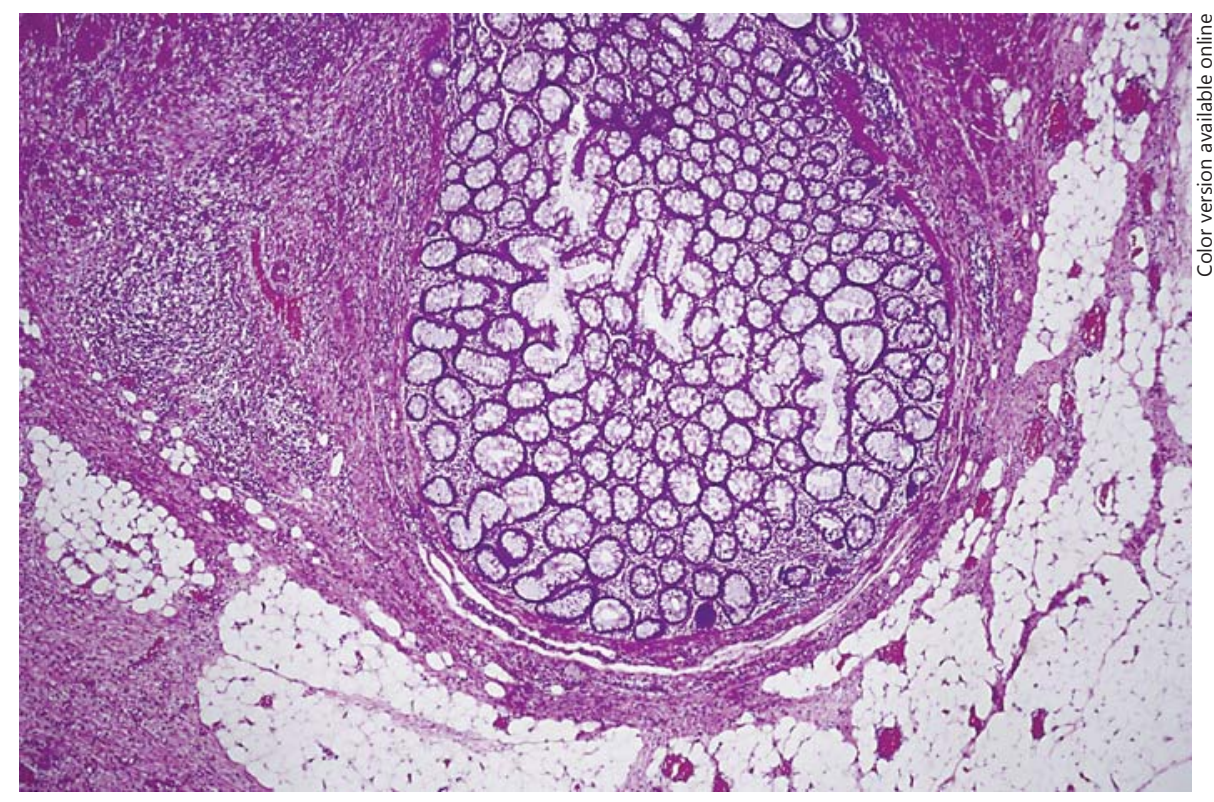

Fig.4. Medium-power image of a left-sided sigmoid diverticulum for comparison. HE. It shows a diverticulum with outpouching of the mucosa and submucosa with an attenuated layer of muscle.

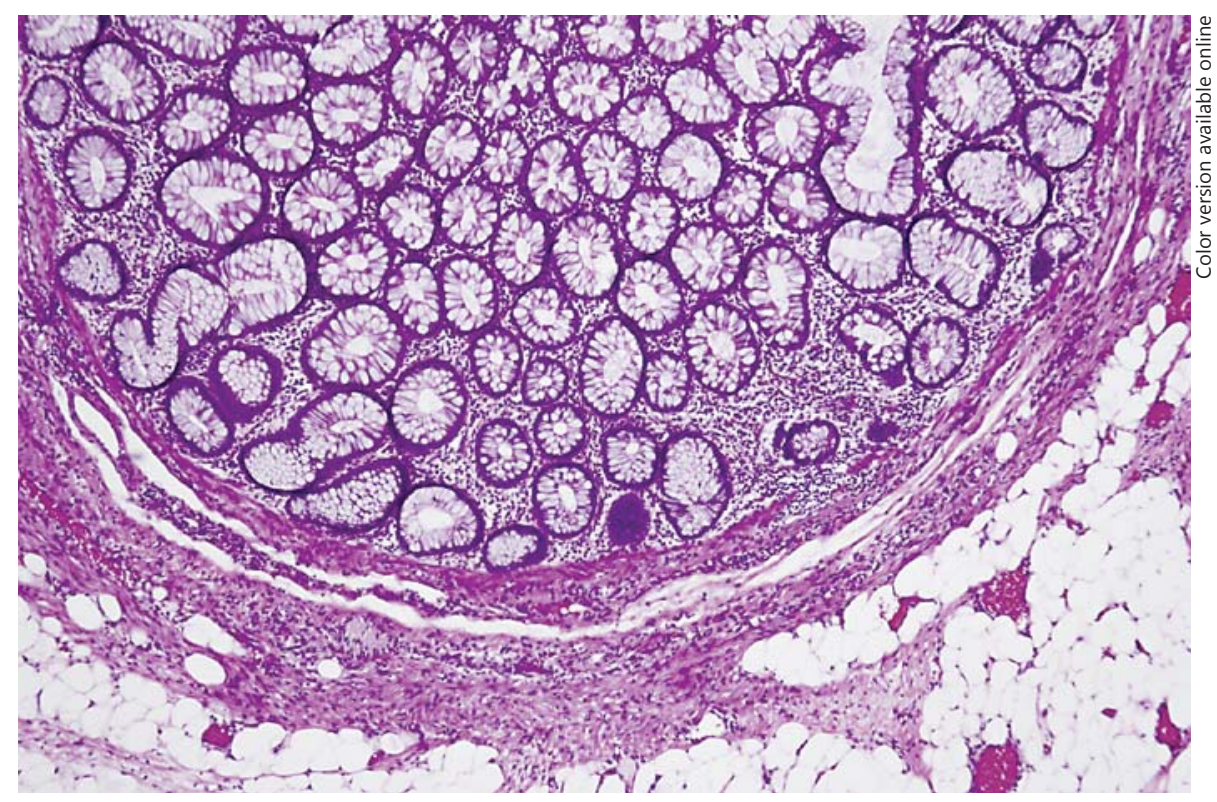

The percentage of RD within the Vietnamese cohort (31\%) was lower than the estimated prevalence of RD in Asians $[16,17]$. This might be attributed to environmental factors such as a Western diet low in fiber and high in fat and red meat, the former of which could lead to a higher rate of $L D$ or LD and RD and the latter two of which have been associated with increased risk of DD of the colon [18]. Correspondingly, our results indicate that a higher percentage of the Vietnamese cohort had LD and $\mathrm{RD}$ than controls (18 vs. $12 \%$ ). Alternatively, as vitamin $\mathrm{D}$ deficiency has also been shown to be associated with a higher risk of hospitalization for diverticulitis [17], geographical effects on the Boston Vietnamese population such as low exposure to sunlight may contribute to the higher rate of diverticulitis and skew the distribution towards LD, although this effect is most likely minimal. Overall, the prevalence of CD in most areas of Asia is low compared to Europe and the USA. However, in westernized urban centers such as Tokyo there is an increasing incidence of $\mathrm{CD}$, of which $\mathrm{RD}$ is most common.

Symptomatic differences between RD and LD have been documented in previous studies, with $\mathrm{RD}$ largely be- 
ing associated with bleeding (50-90\%) and LD with symptoms of diverticulitis and perforation [19]. However, our results showed a similar symptom distribution between LD and RD in both groups, with a higher percentage of bleeding in the left-sided control group (82\%). Interestingly, the Vietnamese cohort had a higher rate of symptoms than the control group, both overall (36 vs. $25 \%$ ) and within the subset of patients with $\mathrm{RD}$ (33 vs. $22 \%)$. Although structural differences in the bowel wall due to genetics must be considered, the fact that more Asians have symptomatic CD is likely to be reflective of the fact that this population is less compliant with screening protocols in the absence of symptoms. Indeed, Vietnamese patients were on average 6 years older than nonVietnamese patients when they presented for their first screening colonoscopy. Thus, educating this population about the importance in this screening method should be emphasized.

In terms of other colonoscopy findings, the rate of hemorrhoids was higher in the Vietnamese population, which may reflect the higher prevalence of irritable bowel syndrome and constipation in the Asian population [20]. Longer length of the transverse and sigmoid colon has been correlated with high rates of constipation in the Japanese, which is thought to result from decreased motility in these predominantly left-sided locations. It is postulated that this could account for increased intraluminal pressure in the right colon, thereby increasing the rate of $\mathrm{RD}$ in the Japanese [21]. Although the Vietnamese population has not been evaluated for colonic length, this structural difference may be another contributing factor to $\mathrm{RD}$ in Vietnamese patients. Consequently, hemorrhoids should be high on the differential diagnosis as the etiology of patients presenting with lower GI bleeding in this population.

The etiology of CD is debatable. However, the most well-known and acceptable pathogenesis involves two mechanisms which act synergistically in the formation of diverticula - increased intraluminal pressure due to a lack of dietary fiber and increased fragility of the colon wall due to raised intraluminal pressure [21]. This also accounts for the increasing incidence of $\mathrm{CD}$ with age, as deposition of immature collagen with abnormal crosslinking creates points of weakness in the colon wall, thereby increasing susceptibility to diverticula. Historically, isolated RD was thought to be congenital and differentiated from LD because all layers of the colon wall are affected [22]. Although genetic factors are likely to play a role in the development of $\mathrm{RD}$, our investigation yielded no gross or histological differences between the two, suggesting that RD could have the same etiology as LD.

Limitations of our study include those inherent in any retrospective cohort study. Selection bias was minimized given our randomized selection of both cohorts, but our dependence on the completeness and accuracy of patient records may have predisposed us to information bias. Moreover, there was frequently an absence of data on potential confounding factors. However, this study is the first, to the best of our knowledge, to evaluate the prevalence, clinical features and associations between $\mathrm{LD}$ and $\mathrm{RD}$ in the Vietnamese population and may open the door for future research in a larger cohort to elucidate the true prevalence, behavior, risk factors, and association of CD in this population. Future studies should aim at assessing all $\mathrm{CD}$-associated variables such as diet, physical activity, smoking status, vitamin D levels, colonic manometry, and changes in enteric/myenteric neuronal regulation of colonic motility, as well as histopathological changes in diverticula-associated blood vessels and tissue adjacent to diverticula. Moreover, identifying when patients moved from their home country to the USA may predict other environmental factors associated with RD.

\section{Conclusion}

In this study $\mathrm{RD}$ was common in the Vietnamese population living in Boston, Mass., USA, and its prevalence was higher in Vietnamese than in Caucasians.

References

1 Etzioni DA, Mack TM, Beart RW Jr, et al: Diverticulitis in the United States: 1998-2005: changing patterns of disease and treatment. Ann Surg 2009;249:210-217.

2 Warner E, Crighton EJ, Moineddin R, et al: Fourteen-year study of hospital admissions for diverticular disease in Ontario. Can J Gastroenterol 2007;21:97-99.

-3 Kang JY, Hoare J, Tinto A, et al: Diverticular disease of the colon - on the rise: a study of hospital admissions in England between 1989/1990 and 1999/2000. Aliment Pharmacol Ther 2003;17:1189-1195.

4 Lee YS: Diverticular disease of the large bowel in Singapore. An autopsy survey. Dis Colon Rectum 1986;29:330-335.

$\checkmark 5$ Roberts P, Abel M, Rosen L, et al: Practice parameters for sigmoid diverticulitis. The Standards Task Force American Society of Colon and Rectal Surgeons. Dis Colon Rectum 1995; 38:125-132. 
6 Richter S, vd Linde J, Dominok GW: Diverticular disease. Pathology and clinical aspects based on 368 autopsy cases (in German). Zentralbl Chir 1991;116:991-998.

7 Petruzziello L, Iacopini F, Bulajic M, et al: Review article: uncomplicated diverticular disease of the colon. Aliment Pharmacol Ther 2006;23:1379-1391.

-8 Vernava AM 3rd, Moore BA, Longo WE, et al: Lower gastrointestinal bleeding. Dis Colon Rectum 1997;40:846-858.

9 Zuckerman GR, Prakash C: Acute lower intestinal bleeding. Part II. Etiology, therapy, and outcomes. Gastrointest Endosc 1999;49: 228-238.

10 Ludeman L, Shepherd NA: What is diverticular colitis? Pathology 2002;34:568.

11 Fenoglio-Preiser CM: Gastrointestinal $\mathrm{Pa}$ thology: An Atlas and Text. Philadelphia, Lippincott Williams, 2008.
12 Fong SS, Tan EY, Foo A, et al: The changing trend of diverticular disease in a developing nation. Colorectal Dis 2011;13:312-316.

13 Miura S, Kodaira S, Shatari T, et al: Recent trends in diverticulosis of the right colon in Japan: retrospective review in a regional hospital. Dis Colon Rectum 2000;43:1383-1389.

14 Hoe J: A radiological survey of diverticulosis in Singapore. Singapore Med J 1991;32:218-220.

15 Arfwidsson S, Knock NG, Lehmann L, et al: Pathogenesis of multiple diverticula of the sigmoid colon in diverticular disease. Acta Chir Scand Suppl 1964;63(suppl 342):1-68.

16 Chia JG, Wilde CC, Ngoi SS, et al: Trends of diverticular disease of the large bowel in a newly developed country. Dis Colon Rectum 1991;34:498-501.

17 Maguire LH, Song M, Strate LE, et al: Higher serum levels of vitamin D are associated with a reduced risk of diverticulitis. Clin Gastroenterol Hepatol 2013;11:1631-1635.
18 Aldoori WH, Giovannucci EL, Rimm EB, et al: Prospective study of diet and the risk of symptomatic diverticular disease in men. Am J Clin Nutr 1994;60:757-764.

19 Wong SK, Ho YH, Leong AP, et al: Clinical behavior of complicated right-sided and leftsided diverticulosis. Dis Colon Rectum 1997; 40:344-348.

20 Gwee KA, Wong RK: Is the constipated Asian male more common than we think? J Gastroenterol Hepatol 2007;22:1168-1169.

21 Nakaji S, Danjo K, Munakata A, et al: Comparison of etiology of right-sided diverticula in Japan with that of left-sided diverticula in the West. Int J Colorectal Dis 2002;17:365373.

22 Morson BC: The muscle abnormality in diverticular disease of the colon. Proc R Soc Med 1963;56:798-803. 